\title{
Scar and Scarring in the Elderly
}

Hester Colboc and Sylvie Meaume

\section{Contents}

44.1 Introduction -380

44.2 Epidemiology of the Elderly: A Factor to Consider - 380

44.3 Definition of Elderly in Medicine: Should We Make Distinctions? - 380

44.4 From Skin Aging to Dermatoporosis - $\mathbf{3 8 0}$

44.5 Consequences of Age on Wound Healing - 381

44.6 Frequent Comorbidities Altering Wound Healing in the Elderly - 381

44.7 What Scarring Problems Are Usually Observed in the Elderly? - 382

44.8 Conclusion -383

References -383 
Aims of the Chapter

- To provide demographic data on the elderly and the definition of elderly in medicine

- To bring elements of skin aging and dermatoporosis

- To describe the consequences of age on healing and major comorbidities in elderly that impair healing

- To address the main problems raised by scars in the elderly - these scars are not the hypertrophic scar, the keloids, or the scars due to cosmetic aspects but the recurrence of a wound on a scar, the Marjolin's ulcer, the hyperkeratosis, the unstable scars, and the adhesion to the deep plane of old scars

\subsection{Introduction}

The population is getting older all over the world. This means that more people are living with chronic illnesses, which reduce their independence and force them to take various medications. Healthcare professionals should take this into account when managing scar and scarring problems in older patients. Until now, research works primarily focus on the treatment and prevention of wounds in the elderly - acute (dissecting hematoma, skin tears, etc.) or chronic (pressure ulcers, leg ulcers, diabetic wounds of the foot, etc.) - but too little was done concerning the problem of scarring itself and even less on scars associated with the loss of skin function along the time.

\subsection{Epidemiology of the Elderly: A Factor to Consider}

According to the United Nations' report on the aging of the world population, adults over 60 years will outnumber younger people by 2050 [1], and in Europe alone, more than $20 \%$ of the population will be over 65 years in 2025 [2]. As this population ages, many older people will live with chronic conditions, reduced independence, polypathology, and polypharmacy, a situation healthcare professionals should take into consideration when dealing with dermatological, scarring, and scar conditions. While governments encourage the growing trend in Europe to promote an adapted elderly management, the specific wound healing and scarring care provided by surgeons, physicians, and caregivers is increasingly important but scarcely discussed [3].

\subsection{Definition of Elderly in Medicine: Should We Make Distinctions?}

If the WHO defines elderly as over 65 years of age, the increase in life expectancy in good health means that when we talk about elderly people in the medical envi-
- Table 44.1 Main factors of frailty in the elderly

Age over 85 years

Loss of autonomy for an activity of daily life or more

Impairment of cognitive functions

Reduced nutritional reserves

Inadequate social support (loneliness, poverty)

Sedentary lifestyle, confinement, postural instability

Poorly compensated sensory disturbances

Kidney and liver failure

Depressive symptoms

Polymedication

ronment, these are subjects of more than 75-80 years, and the distinction between the healthy elderly and the polypathological elderly becomes important. The state of health of the elderly is multifactorial. It depends on genetic, environmental, social, psychic factors, and the existence of multiple pathologies. It is not necessarily linked to age. For 30 years the concept of fragile old man/woman has been developed [4]. It obeys to several definitions and criteria which vary accordingly to the numerous publications of which it is the subject and makes it a dynamic medical concept. Only $10-20 \%$ of the nonagenarians or centenarians are considered fragile. These are the so-called geriatric patients who require specific management. Most people over the age of 75 are not frail and considered "fit" with good functional autonomy and good social integration and few pathologies. Conversely, the frail elderly present functional limitations and a decline in their ability to adapt and anticipate. To distinguish these two types of elderly population, standardized geriatric assessments have been developed using validated scales of autonomy, nutrition, cognition, etc. [5]. This evaluation is not only performed by geriatrician if one wishes exhaustive but can also be realized by nonspecialists and paramedics: nurses, physiotherapists, social workers, dieticians, occupational therapists, and speech therapists. The objective of such assessments is to provide a supportive plan adapted to each patient, a compromise between what is desirable and feasible or reasonable and what is not (• Table 44.1).

\subsection{From Skin Aging to Dermatoporosis}

Skin aging leads to alteration of the skin and consequently to the loss of its physiological barrier role. Skin aging leads to alteration of the skin and consequently to the loss of its physiological barrier role. It is the consequence of intrinsic (genetically programmed) and 


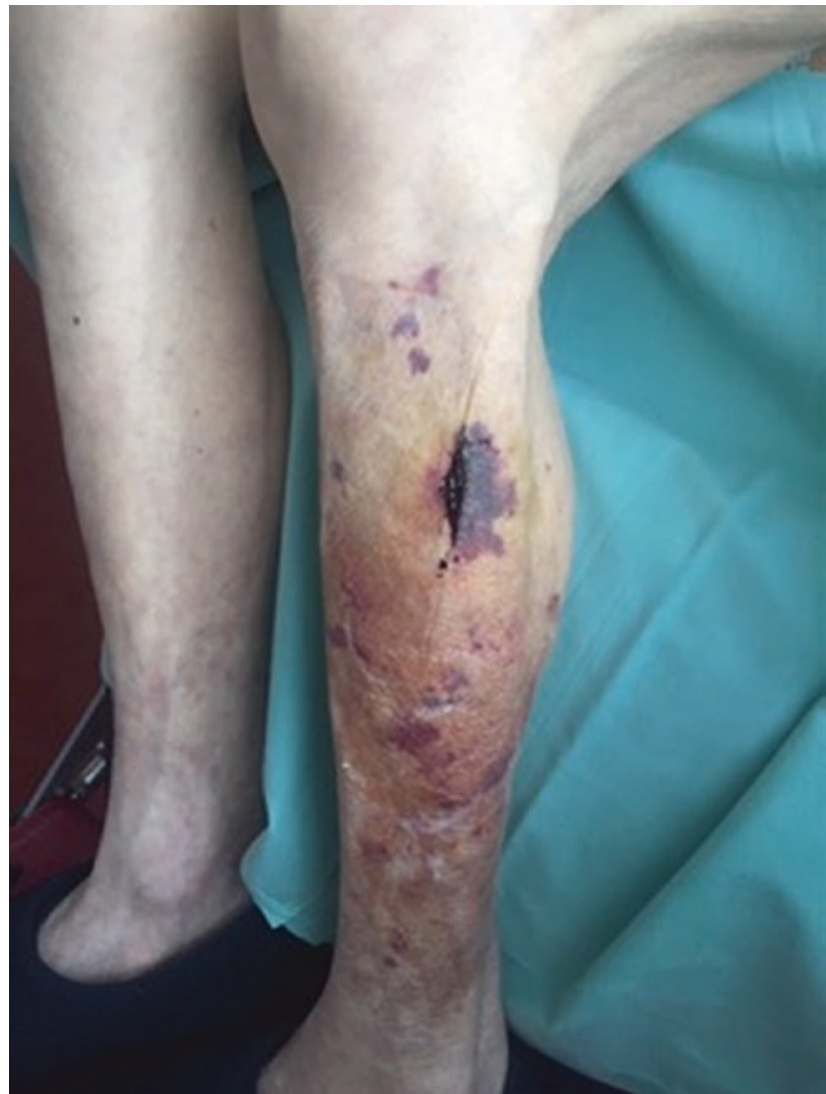

- Fig. 44.1 Dermatoporosis: Bateman purpura, skin tears, hyperpigmentation, and spontaneous stellar pseudo-scars

extrinsic factors. These extrinsic factors are numerous, and include more specifically drugs aggravating the physiological xerosis (hypolipidemic oral, allopurinol, hydroxyurea and cimetidine) [6], deficits in essential fatty acids, in vitamins (B group vitamins, E, PP, C, A), zinc, magnesium as well as malnutrition frequent in the elderly patient and environmental factors such as smoking and exposure to ultraviolet (UV). For some patients, skin aging can become can constitute a real functional organ failure, far from cosmetics considerations. The term "dermatoporosis" was recently proposed by Saurat $[7,8]$ to cover all the manifestations and implications of this chronic skin syndrome linked to skin fragility and insufficiency.

The clinical manifestations of dermatoporosis (- Fig. 44.1) include morphological markers of fragility (skin atrophy, Bateman's purpura, spontaneous stellar pseudo-scars, hyperpigmentation), as well as the functional expression of skin fragility (skin tears, poor healing, dissecting hematoma). The first signs appear around the age of 60 , while the disease itself, with its related complications, is observed between the ages of 70 and 90 .

On a microscopic scale, skin aging is manifested by a thinning of the dermo-epidermal junction and an extension of the keratinocyte renewal time. In the dermis, there is observed a decrease in the number of fibroblasts and thus the density of fiber collagen and glycosaminoglycans, with thinning of the dermal thickness. Associated with this quantitative drop is a qualitative drop in residual fibers. During aging, a decrease of hydrophilic character of the glycosaminoglycans, responsible for the hydration of the dermis, is observed. This chemical modification can explain the dermal thinning and xerosis seen in the elderly. These changes also affect the microvascularization, with thinning of the vessel wall, which may explain the tendency to hematoma and purpura (Batman's purpura) typically observed in the elderly.

\subsection{Consequences of Age on Wound Healing}

Physiological healing has three main stages, directly impacted by aging: the first stage is hemostasis and inflammation, second stage is cell proliferation, and third stage is tissue remodeling. With age, platelet adhesion to the damaged endothelium is increased, decreasing the healing time. The local inflammatory answer to injury is reduced, following a decrease in the expression of molecules of adhesion. The proliferation phase is also impaired by slowing the renewal keratinocyte evokes above and also a decrease in response to growth factors. Finally, tissue remodeling, the final stage of wound healing, is also impaired in the aging subject due to an imbalance between metalloproteinases and their physiological inhibitors, in favor of metalloproteinases and therefore the destruction of collagen [9]. The different healing phases are therefore altered by these phenomena in the elderly: reduction of the wound contraction, cell proliferation, neovascularization, delay of the inflammatory phase, and slower epithelialization [10].

Apart from these deleterious aspects, elderly healing presents some advantages. Thanks to a decrease in inflammation, pathological scarring such as hypertrophic scars and/or keloids is rarely observed in elderly. After skin excision for tumor, sometimes needing a consistent loss of substance, the aged patient skin laxity generally allows an easy reapproximation of the edges and the suture may be realized without tension, issuing to good scarring. However, suturing itself can be impacted by the increased skin tears risks due to the dermatoporosis.

\subsection{Frequent Comorbidities Altering Wound Healing in the Elderly}

Many comorbidities frequent in the elderly can decrease wound healing. Undernutrition and diabetes can delay all type of wound, while arterial or venous diseases and edema (linked to heart, kidney or liver failure) can delay healing of lower limbs wounds. Elderly patients are also more 
exposed to treatments that can delay healing, especially systemic corticosteroid therapy and cancer chemotherapy.

\subsection{What Scarring Problems Are Usually Observed in the Elderly?}

Traumatic and surgical acute wound healing is rather good even in very old patients, and hypertrophic or keloid scars are rare, at least in the Caucasian population [11].

The poor quality of the scar is usually not a major problem (appearance, color, shape) [12] except in exposed regions (face) or when impacting the function (heel, eyelid, and periorificial areas).

Pruritus and/or pain may appear very lately after the trauma/surgery and occur after 20 or 30 years. They are linked to dermatological problem (dry skin) or neurogenic disorders. These situations may be treated symptomatically with appropriate cosmetics [13]. A few of them need surgery, but war, posttraumatic, and postsurgery scars are not usually reasons to go to see a doctor.

Atrophic and adherent scars can be improved by injecting fat under the scar, a recent technique presenting the advantage of being scarcely invasive when anticoagulation is not needed or should be stopped (- Fig. 44.2).

The reappearance of a wound on a scar should be considered differently. The recurrence of a tumor may be at the origin of the scar. A biopsy or the recurrence of a chronic ulcer (arterial or venous, pressure, or diabetic foot ulcers) is needed to diagnose a malignant transformation.

It can also lead to the reassessment of the patient and indication of preventive treatment of compression or discharge (cushion, shoe, soles).

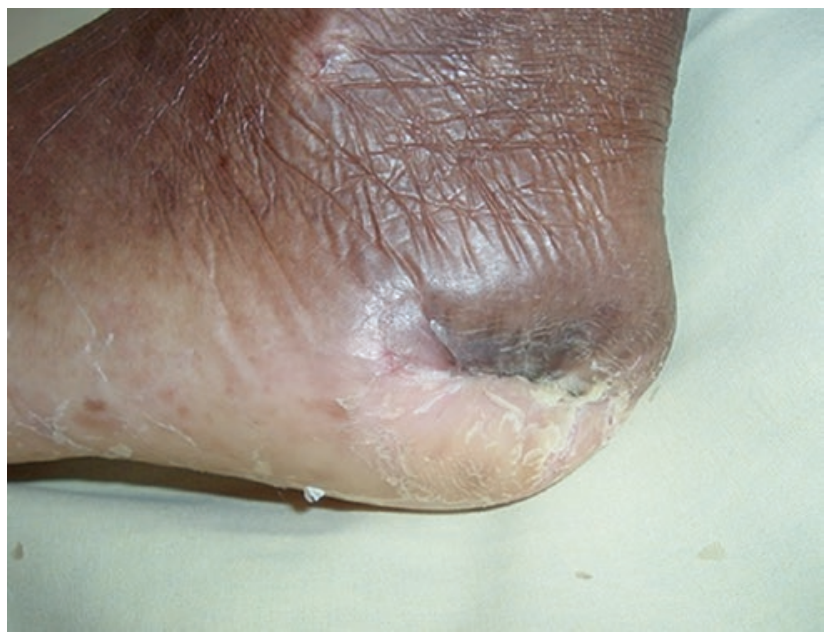

- Fig. 44.2 Atrophic scar/defect: In this stage 4 pressure ulcer, the healing process led to a neoskin adherent to the underlying structures like aponeurosis, muscle, tendon, and bone. The possibility to inject adipose tissue under the skin should be discussed
Post-irradiation scars in cancer treatments (breasts for example) pose the problem of radiodermatitis and radionecrosis which evolves and worsens over time, in particular in the elderly who were irradiated at a time when the administered doses were high.

Some scars from childhood linked to operated orthopedic malformations are associated with joint deformations of osteoarthritis because of mechanical forces pressure or friction exerted on those scars or because of underlying medical problems in the region: arterial disease or neuropathy.

Marjolin's ulcer [14], a rare and aggressive skin cancer, develops late on scars from burns (- Fig. 44.3) or from delayed wound-healing problems: chronic osteitis, burns, pressure ulcers, lupus scar, skin graft, and radiodermatitis. Squamous cell carcinoma are more frequently observed than basal cell carcinoma, melanoma, or sarcoma. The occurrence of a wound on a scar aged of more than 20 years always requires a biopsy [15].

Hyperkeratosis is common especially on scars on plantar aspect of the foot, the heel, next to the Achilles tendon, or on the toes and the lateral aspects of the foot, due to a thickening of the stratum corneum reaction to friction or to mechanical conflict, especially in case of loss of sensibility (diabetes, nerve damage). Cracks can appear and constitute entry doors exposing to the risk of deep infection (• Fig. 44.4).

Unstable scars may appear because of their location, sometimes due to a poor quality of the dermal component (absent or fibrotic) or insufficient preventive measures taken against external agents (shoes, stockings, bandages, prosthesis, etc.).

These wounds finally closed after a succession of closure and multiple reopenings, source of discomfort, and risk of cancer transformation (- Fig. 44.5). The treatment of these unstable scars becomes more and more complex due to the underlying diseases and comorbidi-

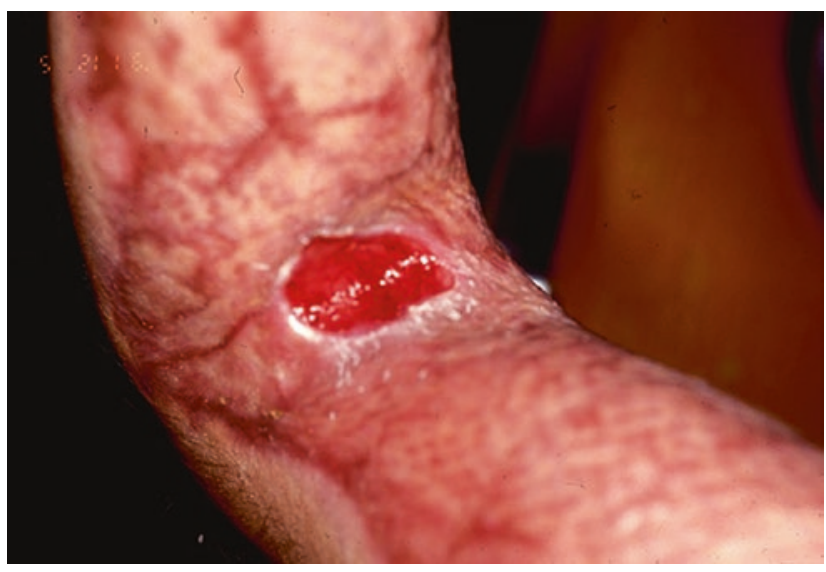

- Fig. 44.3 Marjolin's ulcer on an old burn scar 


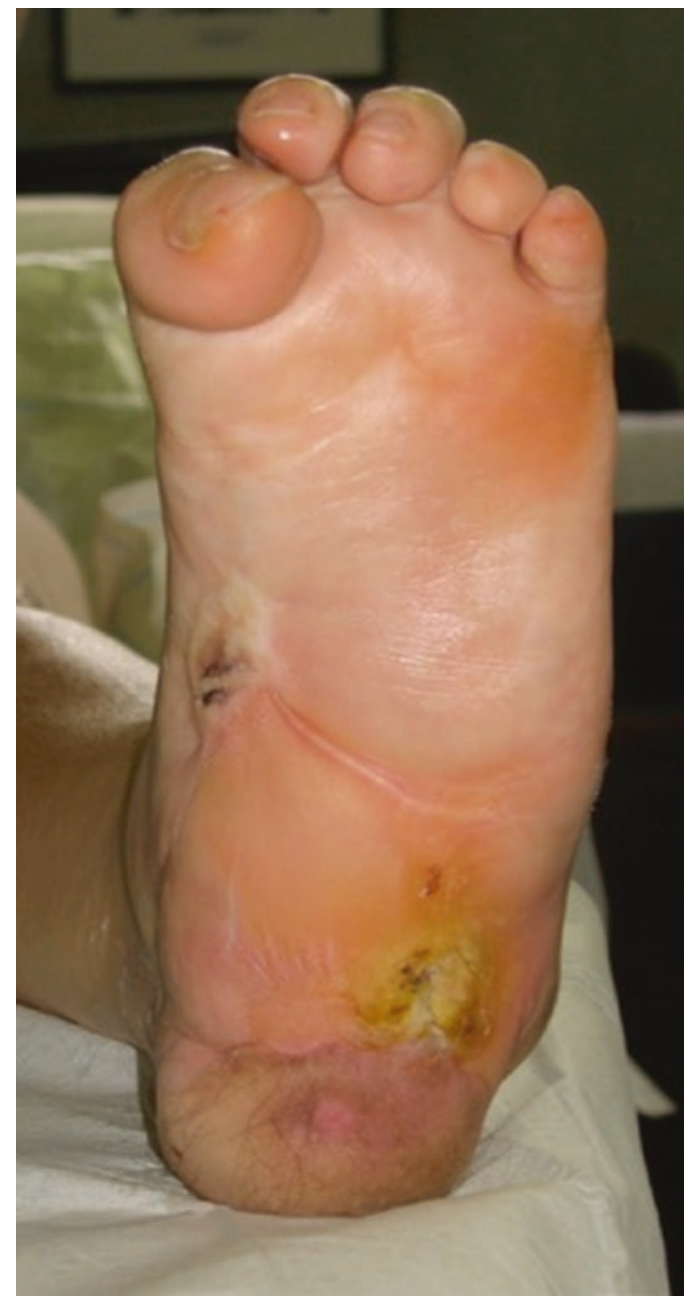

- Fig. 44.4 Scar becoming problematic with the age due to mechanical forces, trauma, pressure over the scar, underlying pathology (osteitis, diabetes, vascular diseases venous or arterial) issuing to hyperkeratosis, and potential neoplasic transformation

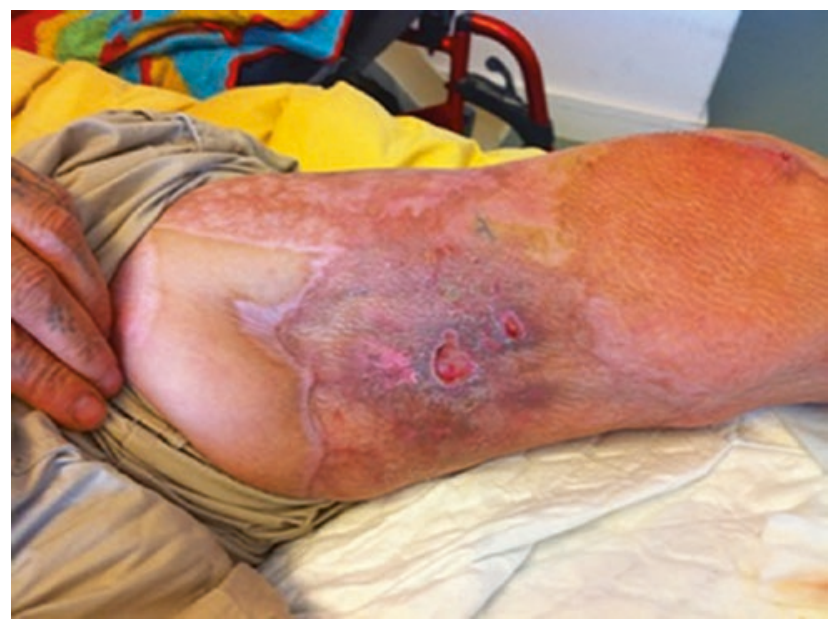

- Fig. 44.5 Scar instability: consequence of poor quality of dermal component (absent or fibrotic) and poor preventive measures taken against external mechanical agent (shoes, stocking, bandage, prosthesis, etc.). Succession of closure and reopening phases ties. Biopsies often need to be done to rule out a malignant degenerescence.

Adherence to the depth is a problem that worsens with age. Healing occurring on a wound with loss of deep substance (dermis, fat, gliding capacities) and not correctly repaired (for exemple using negative pressure therapy, skin substitute or flap) may lead to an atrophic scar adherent to the deep plans. These adherent scars can disrupt normal skin movements and mobilisation of the patient, specially when located near an articulation. These adherences are exposed to skin reopening due to the loss of elasticity of age-related skin.

\subsection{Conclusion}

Epidemiological studies are lacking and the literature is poor on the subject. Hypertrophic and keloids do not seem to be a frequent problem in the elderly. The absence of inflammation and tension on the scar is undoubtedly an explanatory factor that perhaps merits more basic research. However, persistent functional problems lead aged patients to consult. Chronic wounds or sites of a long-lasting healing process are associated to an increased risk of malignant transformation, that must be take into consideration by the clinician. Regarding scars, elderly patients are usually less concern by the cosmetic aspects and its impact on the quality of life. The existence of underlying diseases can reopen certain scars or pressure ulcers and therefore the treatment must be adapted according to the comorbidities. The geriatrician sometimes has a role to play in establishing the therapeutic plan.

\section{Take-Home Messages}

- The number of elderly people is increasing.

- The tension on the scars is often less because the skin of the elderly is less elastic (modification of the extra cellular matrix).

- Keloid and hypertrophic scars are rare in the elderly.

- Scars problems are more often malignant degenerescence, hyperkeratosis, atrophy, instability, or adherence to the depth.

- A biopsy must be performed if the scar reopens or persists in an elderly patient.

\section{References}

1. United Nations. World population ageing: 1950-2050. New York: United Nations; 2001.

2. European Commission. Ageing. Available from: http://ec.europa.eu/ health/ageing/policy/index_en.htm. Accessed December 2014. 
3. Rechel B, Doyle Y, Grundy E, McKee M. Policy brief 10: how can health systems respond to population ageing? vol. 36 . Geneva: World Health Organization; 2009.

4. Buchner DM, Wagner EH. Preventing frail health. Clin Geriatr Med. 1992;8(1):1-17.

5. Dent E, Kowal P, Hoogendijk EO. Frailty measurement in research and clinical practice: a review. Eur $\mathbf{J}$ Intern Med. 2016;31:3-10.

6. Greist MC, Epinette WW. Cimetidine-induced xerosis and asteatotic dermatitis. Arch Dermatol. 1982;118:253-4.

7. Saurat JH. Dermatoporosis - the functional side of skin aging. Dermatology. 2007;215:271-2.

8. Kaya G, Saurat JH. Dermatoporosis: a chronic cutaneous insufficiency/fragility syndrome. Clinicopathological features, mechanisms, prevention and potential treatments. Dermatology. 2007;215:284-94.

9. Quan T, Fisher G. Role of age-associated alterations of the dermal extracellular matrix microenvironment in human skin aging: a mini-review. Gerontology. 2015;61(5):427-34.
10. Sgonc R, Gruber J. Age-related aspects of cutaneous wound healing: a mini-review. Gerontology. 2013;59:159-64.

11. Monarca C, Maruccia M, Palumbo F, Parisi P, Scuderi N. A rare case of postauricular spontaneous keloid in an elderly patient. In Vivo. 2012;26(1):173-5.

12. Brands-Appeldoorn A, Maaskant-Braat S, Zwaans W, Dieleman J, Schenk K, Broekhuysen C, Weerdenburg H, Daniel R, TjanHeijnen V, Roumen R. Patient-reported outcome measurement compared with professional judgment of cosmetic result afeter breast-conserving therapy. Curr Oncol. 2018;25(6):553-61.

13. Humbert P, Dréno B, Krutmann J, Luger AT, Triller R, Meaume $\mathrm{S}$, Seité S. Recommendations for managing cutaneous disorders associated with advancing age. Clin Interv Aging. 2016;11: $141-8$.

14. Cruickshank AH, Gaskele E. Jean-Nicolas Marjolin: destined to be forgotten? Med Hist. 1963;7:383-4.

15. Yu N, Long X, Lujan-Hernandez JR, et al. Marjolin's ulcer: a preventable malignancy arising from scars. World J Surg Oncol. $2013 ; 11: 313$.

Open Access This chapter is licensed under the terms of the Creative Commons Attribution 4.0 International License (http://creativecommons. org/licenses/by/4.0/), which permits use, sharing, adaptation, distribution and reproduction in any medium or format, as long as you give appropriate credit to the original author(s) and the source, provide a link to the Creative Commons license and indicate if changes were made.

The images or other third party material in this chapter are included in the chapter's Creative Commons license, unless indicated otherwise in a credit line to the material. If material is not included in the chapter's Creative Commons license and your intended use is not permitted by statutory regulation or exceeds the permitted use, you will need to obtain permission directly from the copyright holder. 